\title{
Multimodal multidetector computed tomography scanning and the validation of a standardized protocol
}

\author{
Avaliação multimodalidade por meio da tomografia computadorizada multidetector: \\ validação de um protocolo padronizado
}

Claudia Costa Leite

Professora Associada do Departamento de Radiologia

da Faculdade de Medicina da

Universidade de São Paulo

(FMUSP); Chefe do LIM44 do

Hospital das Clínicas da FMUSP.

São Paulo SP, Brasil.

Correspondence

Claudia Costa Leite

Instituto de Radiologia, Setor de

Ressonância Magnética

Rua Dr. Enéas de Carvalho Aguiar

$255 /$ portaria $5 / 3^{\circ}$ andar

05403-001 São Paulo SP - Brazil

E-mail: claudia.leite@hc.fm.usp.br

Conflict of interest

There is no conflict of interest to

declare.

Received 17 April 2013

Accepted 24 April 2013
I schemic stroke is a common and serious disorder. Worldwide, stroke is a leading cause of death and disability. In the United States, the incidence is of about 795,000 each year ${ }^{1}$. In Brazil, stroke is a leading cause of mortality, and age-adjusted mortality rates for stroke are higher than in other South American countries².

In the recent years, the development of new therapeutic techniques for hyperacute ischemic stroke has created a prompt need of a more precise diagnosis of this entity ${ }^{3}$. As well as the identification of ischemic lesions, the definition of the extent and location of the infarct are also key factors in the therapeutic decisions. Non-contrast computed tomography (CT) can confidently rule out a hemorrhagic stroke, but in a great percentage of patients it can be a false negative in the detection of lesions in the first hours of stroke ${ }^{1}$. In contrast, magnetic resonance imaging (MRI) is a reliable tool to detect cytotoxic brain edema. Perfusion sequences allow detection of mismatch areas, and MR-angiography detects arterial flow abnormalities. However, MRI is not always available in all emergency units and it has higher costs than CT. Multimodal CT has also been increasingly used for this purpose, being a very fast and reliable imaging examination, very adequate for an emergency situation ${ }^{4}$.

The paper in this issue of Arquivos de Neuropsiquiatria: "Multiparametric multidetector computed tomography scanning of hyperacute ischemic stroke: validating a standardized protocol" by Pacheco et al. ${ }^{5}$ suggests a multimodal protocol for hyperacute ischemic stroke including non-contrast CT, CT-perfusion and CT-angiography (CT-angio). This paper also presents an interesting step-by-step orientation for the interpretation of these exams, as well as the role of the reader experience in the final detection of ischemic stroke.

The development of multidetector CT equipments allowed that CT-perfusion and CT-angio could be performed easily in a few minutes. The association of information of these different modalities of CT (also called multimodal CT imaging or multiparametric multidetector CT scanning) has been increasingly used for acute stroke imaging. The rationale of the use of multimodal CT scanning is to rule out or demonstrate hemorrhagic stroke or clearly visible large infarcts (nonenhanced CT), to define the location and extent of ischemia and to characterize salvageable brain tissue (CT-perfusion), as well as to assess for a possible underlying vessel occlusion or other vascular pathology (CT-angio).

Multimodal CT imaging has been proven to allow a more precise diagnosis of hyperacute stroke 6 . In the paper from Pacheco et al. ${ }^{5}$, there was no false negative diagnoses of acute ischemic stroke when the different CT techniques using a standardized reading protocol were associated. The definition of a standardized step-by-step interpretation in multiparametric multidetector CT in the evaluation of the signs of hyperacute stroke can aid in the detection of subtle signs of this type of lesion. 
The discrepancy that this paper found between the different backgrounds in imaging evaluation (general Radiology residents versus Neuroradiology residents) reinforces the necessity of a specific training program to interpret multiparametric examinations precisely. The interobserver agreement between Neuroradiology fellows was higher than between the general Radiology residents ${ }^{5}$.

The paper also presents an important point to be discussed: the need of an adequate post-processing of CT perfusion and CT-angio. These post-processing algorithms, when properly used, allow obtaining the best information from all methods. Inadequate post-processing can obscure subtle lesions ${ }^{6}$.

At last, it is important to mention that, even though multimodal CT could be done fast and easily with multidetector CT equipments, radiation dose should be a permanent concern. Although research into protocol design and newer CT scanner technologies enable high-quality examinations to be performed with a significant reduction in radiation dose ${ }^{7}$, this point should be always kept in mind in future CT studies.

\section{References}

1. Latchaw RE, Alberts MJ, Lev MH, et al. American Heart Association Council on Cardiovascular Radiology and Intervention, Stroke Council, and the Interdisciplinary Council on Peripheral Vascular Disease. Recommendations for imaging of acute ischemic stroke: a scientific statement from the American Heart Association. Stroke 2009;40:3646-3678

2. Lotufo PA, Bensenor IM. Trends of stroke subtypes mortality in São Paulo, Brazil (1996-2003). Arq Neuropsiquiatr 2005;63:951-955.

3. Kirmani JF, Alkawi A, Panezai S, Gizzi M. Advances in thrombolytics for treatment of acute ischemic stroke. Neurology 2012;79:S119-S125.
4. Fisher M, Albers GW. Advanced imaging to extend the therapeutic time window of acute ischemic stroke. Ann Neurol 2013;73:4-9.

5. Pacheco FT, Rocha AJ, Littig IA, et al. Multiparametric meultidetector computed tomography scanning on suspicion of hyperacute ischemic stroke: validating a standardized protocol. Arq Neuropsiquiatr 2013;71:349-356.

6. Frölich AM, Psychogios MN, Klotz E, Schramm R, Knauth M, Schramm P. Angiographic reconstructions from whole-brain perfusion CT for the detection of large vessel occlusion in acute stroke. Stroke 2012;43:97-102.

7. Gunn ML, Kohr JR. State of the art: technologies for computed tomography dose reduction. Emerg Radiol 2010;17:209-218. 